OPEN ACCESS

Edited by:

Maarten Koeners,

University of Bristol, UK

Reviewed by:

Kirk L. Hamilton,

University of Otago, New Zealand

Christoffer Laustsen,

Aarhus University, Denmark

*Correspondence:

Menno Pruijm

menno.pruijm@chuv.ch

Specialty section: This article was submitted to Renal and Epithelial Physiology,

a section of the journal

Frontiers in Physiology

Received: 01 November 2016 Accepted: 19 December 2016

Published: 05 January 2017

Citation:

Pruijm M, Milani B and Burnier M (2017) Blood Oxygenation Level-Dependent MRI to Assess Renal

Oxygenation in Renal Diseases:

Progresses and Challenges.

Front. Physiol. 7:667.

doi: 10.3389/fphys.2016.00667

\section{Blood Oxygenation Level-Dependent MRI to Assess Renal Oxygenation in Renal Diseases: Progresses and Challenges}

\author{
Menno Pruijm *, Bastien Milani and Michel Burnier \\ Service of Nephrology and Hypertension, Department of Medicine, Centre Hospitalier Universitaire Vaudois, Lausanne, \\ Switzerland
}

BOLD-MRI (blood oxygenation-level dependent magnetic resonance imaging) allows non-invasive measurement of renal tissue oxygenation in humans, without the need for contrast products. BOLD-MRI uses the fact that magnetic properties of hemoglobin depend of its oxygenated state:: the higher local deoxyhemoglobin, the higher the so called apparent relaxation rate $\mathrm{R} 2 *\left(\mathrm{sec}^{-1}\right)$, and the lower local tissue oxygen content. Several factors other than deoxyhemoglobin (such as hydration status, dietary sodium intake, and susceptibility effects) influence the BOLD signal, and need to be taken into account when interpreting results. The last 5 years have witnessed important improvements in the standardization of these factors, and the appearance of new, highly reproducible analysis techniques of BOLD-images, that are reviewed in this article. Using these new BOLD-MRI analysis techniques, it has recently been shown that persons suffering from chronic kidney diseases (CKD) have lower cortical oxygenation than normotensive controls, thus confirming the chronic hypoxia hypothesis. The acute alterations in R2* after the administration of furosemide are smaller in CKD, and represent an estimate of the oxygen-dependent tubular transport of sodium. BOLD-MRI-alone or in combination with other functional MRI methods- can be used to monitor the renal effects of drugs, and is increasingly used in the preclinical setting. The near future will tell whether or not BOLD-MRI represents a new tool to predict renal function decline an adverse renal outcome.

Keywords: BOLD-MRI, chronic kidney disease, renal artery stenosis, furosemide, TLCO-technique

\section{INTRODUCTION}

Chronic kidney disease (CKD), defined as an estimated glomerular filtration rate below 60 $\mathrm{ml} / \mathrm{min} / 1.73 \mathrm{~m}^{2}$ and/or the presence of (micro) albuminuria, has become a major public health problem with a global prevalence in the general population of $\sim 10 \%$ (Ponte et al., 2013). CKD is an independent cardiovascular risk factor and associated with increased mortality (Astor et al., 2011). The pathophysiology of CKD and its progression to end stage renal disease is complex and incompletely understood, but mounting evidence from animal studies suggests that renal tissue hypoxia is the final and common pathway, irrespective of etiology (Alberti and Zimmet, 1998; Fine and Norman, 2008). According to this "chronic hypoxia hypothesis," loss of peritubular capillaries induces interstitial hypoxia which triggers local inflammation and fibrosis. This leads in turn to 
further obliteration and loss of capillaries, thus completing the vicious circle. So far, evidence for the chronic hypoxia hypothesis in humans has been sparse, mainly because of the lack of methods to assess renal tissue oxygenation in a reliable, non-invasive manner.

A technique to measure tissue oxygenation in humans would be a valuable tool for several reasons. First of all, such a technique could be used to confirm or reject the chronic hypoxia hypothesis. Secondly, ideally it would allow to identify CKD patients at increased risk for rapid renal function decline and end-stage renal disease, since according to the chronic hypoxia hypothesis, those with the lowest degree of oxygenation have the highest renal risk. Finally, drugs that chronically increase renal tissue oxygenation would have the potential to retard the progression of CKD. Thus, a method capable of measuring renal tissue oxygenation could identify at an early stage drugs with oxygen-increasing and possibly nephroprotective potential.

Since its first description in 1996 (Prasad et al., 1996), renal blood oxygenation-level dependent MRI (BOLD-MRI) is seen by many as the most promising method to assess renal oxygenation non-invasively in humans. In brief, BOLD-MRI uses the paramagnetic properties of deoxyhemoglobin to assess tissue oxygenation: the higher local deoxyhemoglobin, the higher the apparent relaxation rate $\mathrm{R} 2^{*}\left(\mathrm{sec}^{-1}\right)$, and the lower local tissue oxygen content, assuming that blood $\mathrm{pO}_{2}$ is in equilibrium with tissue $\mathrm{pO}_{2}$. BOLD-MRI does not require the administration of (possibly nephrotoxic) contrast media, making it an interesting tool for CKD patients. BOLD-MRI is fast and can be repeated many times in short time intervals without side-effects.

Despite these advantages, BOLD-MRI is for the moment mainly used in research settings and not yet fully integrated in clinical practice. The initial enthusiasm was tempered by studies who failed to demonstrate with BOLD-MRI that chronic hypoxia is indeed present in humans. Besides, we have learned that factors other than oxygenation (such as blood $\mathrm{pH}$, hematocrite, hydration status, and susceptibility effects, see below) influence the BOLD-signal (Prasad and Epstein, 1999; Pruijm et al., 2010; Neugarten, 2012). However, progress has been made in the standardization of these factors, as well as in data acquisition and analysis, which has recently lead to interesting results and will possibly lead to new applications for this technique. In this article, we review the technical hurdles that had to be overcome, others that still need to be resolved, the main results of clinical studies and the perspectives of BOLD-MRI.

\section{BOLD-MRI: TECHNIQUE AND PITFALLS}

The basic principle of Magnetic Resonance Imaging (MRI) can be summarized as follows: when atomic nuclei with non-zero angular momentum (like hydrogen) are placed in a magnetic field and reach thermal equilibrium, an excess of nuclei lying in the low energy state appears. This excess of nuclei can be brought to some higher energy state when excited by an electromagnetic wave (pulse). The nuclei return to equilibrium after the excitation while emitting back a radiation; this takes a certain time, measured as the so called relaxation times (T1 and T2). These parameters provide information about the density and localization of (hydrogen or other) nuclei, and allow the construction of an image.

BOLD-MRI measures the apparent relaxation rate $\mathrm{R} 2 *$ (or decay rate, defined as $1 / \mathrm{T} 2 *$ and expressed in $\mathrm{sec}^{-1}$ ) for each voxel located in the kidney. This parameter is influenced by any kind of inhomogeneity in the static magnetic field, in particular by the effect of deoxyhemoglobin, which has a strong positive magnetic susceptibility due to its central iron atom. The susceptibility difference between deoxyhemoglobin and surrounding tissues will generate intra-voxel magnetic field inhomogeneities. As such, the decay rate $\mathrm{R} 2 *$ will be enhanced when the local deoxyhemoglobin concentration is increased. Assuming that blood pO2 is in equilibrium with tissue pO2, R2* values allows to qualitatively compare tissue oxygenation between different voxel of the same subject and between subjects: low R2* values indicates high tissue oxygenation, and vice versa (Prasad, 2006). BOLD-MRI has been validated in animal studies, showing that $\mathrm{R} 2 *$ correlates negatively with directly measured pO2 (Pedersen et al., 2005). However, several points merit attention in the interpretation of $\mathrm{R} 2{ }^{*}$ values. First, factors that affect the oxygen dissociation curve (which describes the relationship between $\operatorname{de}($ oxy $) \mathrm{Hb}$ and pO2) such as blood $\mathrm{pH}$, body temperature, and hematocrite alter the equilibrium described above, and should be taken into account when performing BOLD-MRI (Neugarten, 2012). Second, the BOLD-signal is sensitive to an acute water load (Prasad and Epstein, 1999). This sensitivity is partly explained by the waterinduced reduction in oxygen-consuming tubular transport, but an increase in tubular volume after water intake will also reduce the local deoxyhemoglobin volume fraction and thus further decrease R2*. Standardization of water intake is therefore warranted. Because tubular sodium reabsorption is a major driver of oxygen consumption, short-term alterations in dietary sodium intake influence significantly medullary R2* values, and urinary sodium excretion (as a proxy of sodium intake) should therefore be measured whenever possible (Pruijm et al., 2010).

While the acquisition of BOLD-MRI is performed in many centers worldwide, no general consensus exists on how to analyze the BOLD-images, and many methods are in use (see Figure $\mathbf{1}$ for a few examples). In the oldest and most frequently used method, the ROI technique, small circles each containing a collection of voxels -called regions of interest (ROI's)- are placed manually in the cortex and in the medulla of each slice (Figure 1, left image). This allows the calculation of the average cortical and medullary R2* values, per kidney or for both kidneys together (Simon-Zoula et al., 2006). Placement of ROIs is easy in kidneys with preserved renal function, but difficult in patients with advanced CKD due to the lack of cortico-medullary differentiation in the latter (e.g., the human eye is no longer capable of differentiating the cortex from the medulla purely based on radiological contrast differences). A second, recent method called the TLCO (12 layers concentric objects) technique is a semi-automatic procedure that divides the kidney in 12 layers of equal thickness. The mean R2* values of all layers can be plotted as a curve (the R2* radial profile) with a certain slope (Figure 1, middle image). The steepness of the slope is associated with the degree of CKD: the higher the eGFR, the steeper the slope (Milani et al., 2016). The R2* profile has a 

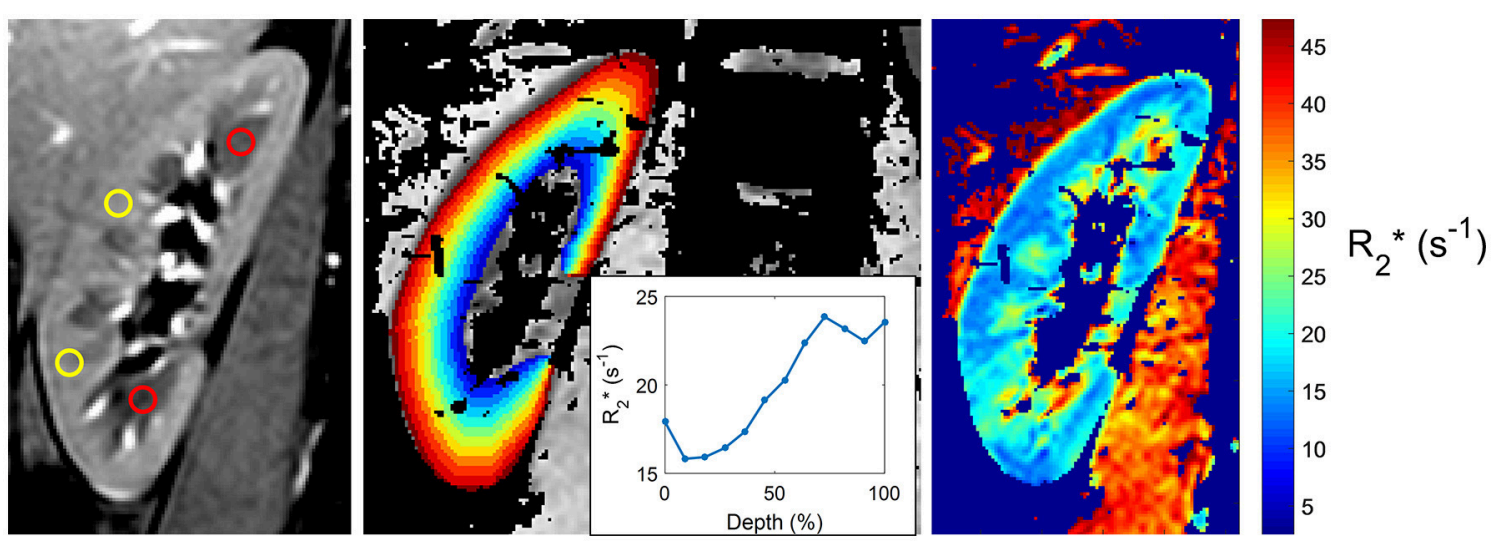

FIGURE 1 | Three currently used techniques to analyze BOLD-MRI. (Left) Classical ROI-based technique, with placement of circle-shaped regions of interest (ROIs) in the renal cortex (yellow) and medulla (red); (Middle) Twelve-Layer Concentric Objects (TLCO) technique, which divides the renal parenchyma in 12 layers of equal thickness; (Right) Fractional hypoxia technique, which counts the percentage of voxels with an $\mathrm{R} 2^{*}$ value $>30$ sec ${ }^{-1}$.

low inter-observer variability and can visualize layer by layer the effect of external stimuli, making it an interesting tool to study the effects of drugs, as recently demonstrated (Vakilzadeh et al., 2015).

Since the BOLD-signal is influenced by many factors, of which some are scanner related, absolute $\mathrm{R} 2 *$ values are not comparable across sites. The steepness of the R2* slopes less of the $\mathrm{R} 2 *$ values, but merely of their intra-compartmental distribution. This observable may therefore be more suitable for comparisons across centers, which would be a huge advantage for eventual future integration in clinical practice. Another way to overcome this hurdle is to perform a dynamic test (such as the administration of IV furosemide or $100 \%$ oxygen breathing), and to assess the percentage change in $\mathrm{R} 2 *$.

In a third method called the fractional tissue hypoxiatechnique, the whole renal parenchyma is selected, and the percentage of $\mathrm{R}^{*}$ values above a certain threshold (usually $30 \mathrm{~s}^{-1}$ or $>2.5$ standard deviations of the average $\mathrm{R} 2^{*}$ value) is reported (Saad et al., 2013). This technique provides a variablethe percentage of hypoxic tissue- that can be easily interpreted by clinicians, but it does not differentiate between cortex and medulla (Figure 1, right image).

Taken together, each technique has his advantages and disadvantages. The most promising and reproducible technique for CKD patients is at the moment, to our opinion, the TLCO method; international efforts are ongoing and necessary to standardize analysis methods in the years to come.

\section{BOLD-MRI IN CKD}

Surprisingly, BOLD-MRI studies have not uniformly demonstrated that renal tissue oxygenation is reduced in CKD patients as compared with controls. Some studies reported higher R2* values suggesting lower renal oxygenation at lower eGFR, whereas other studies did not find any correlation between CKD status and R2* (Table 1). Unfortunately, many of the "early" studies lacked information on drug-or sodium intake, did not standardize water intake, or did not differentiate between the underlying causes of CKD. However, even well-designed studies failed to show differences in R2* between persons with and without CKD (Khatir et al., 2015). It has become clear that the analysis technique has a large impact on the results. Our research group has recently demonstrated that the ROI technique is highly observer-dependent in advanced CKD, and should therefore not be used in these patients (Piskunowicz et al., 2015). The importance of the used analysis technique is further illustrated by the fact that our research group did not find any differences in $\mathrm{R} 2 *$ with the ROI technique, whereas the use of the TLCO technique in the same patient cohort showed significant differences in $\mathrm{R} 2 *$ between the cortical layers of CKD patients and controls (Milani et al., 2016).

As can be appreciated in Table 1, recent BOLD-MRI studies have actually demonstrated that CKD patients have higher R2* values (corresponding to lower renal tissue oxygenation) as compared to controls, thus confirming the findings of animal studies. Differences in R2* were mainly confined to the cortex, which is somewhat surprising, since the cortex has higher pO2 values than the medulla (50 vs. $10-20 \mathrm{mmHg}$ ), receives $90 \%$ of the renal blood flow, and has relatively lower oxygen consumption than the medulla (Aukland and Krog, 1960; Heyman et al., 2008). In theory, the cortex should therefore be better protected against hypoxia. Whether this is explained by glomerular hyperfiltration in residual glomeruli, altered diffusive shunting pathways or reduced metabolic efficiency of solute transport in tubular cells is actually unclear. Of note, although the mean R2* is higher in CKD patients, differences are small, and according to some authors mainly driven by a minority of CKD patients with high R2* values (Prasad et al., 2015). This finding illustrates that renal tissue oxygenation is rather tightly controlled in the majority of individuals. In this respect, some have stated that interstitial fibrosis- the consequence of hypoxia and one of the hallmarks of advanced kidney disease- might be a way to maintain to a certain degree renal oxygenation, by decreasing local oxygen-consuming 
TABLE 1 | Overview of the studies that have used BOLD-MRI to assess renal tissue oxygenation in CKD patients as compared with controls.

\begin{tabular}{|c|c|c|c|c|c|c|c|c|}
\hline Author & & $\boldsymbol{N}$ & Design & Field strength (Tesla) & Analysis method & R2* cortex & $\mathrm{R}^{*}$ medulla & Remark \\
\hline Inoue & 2011 & 119 & CKD-control & 1.5 & $\mathrm{ROI}$ & No difference & Not available & Only DM \\
\hline Wang & 2011 & 27 & CKD-control & 1.5 & $\mathrm{ROI}$ & No difference & Lower in CKD & Only DM \\
\hline Michaely & 2012 & 280 & Observational & 1.5 and 3 & $\mathrm{ROI}$ & No difference & No difference & \\
\hline Xin-Long & 2012 & 26 & CKD-control & 3 & $\mathrm{ROI}$ & Higher in CKD & Higher in CKD & \\
\hline Yin & 2012 & 115 & CKD-control & 3 & $\mathrm{ROI}$ & Higher in CKD & Higher in CKD & Only DM \\
\hline Thacker & 2015 & 47 & CKD-control & 3 & Large ROI-entire parenchyma & Higher in CKD & No difference & \\
\hline Prasad & 2015 & 59 & CKD-control & 3 & $\mathrm{ROI}$ & Higher in CKD & No difference & \\
\hline Khatir & 2015 & 86 & CKD-control & 1.5 & $\mathrm{ROI}$ & No difference & No difference & \\
\hline Milani & 2016 & 207 & CKD-control & 3 & TLCO & Higher in CKD & Lower in CKD & \\
\hline
\end{tabular}

active transport. Long-term 5/6 nephrectomy models in the rat have indeed reported maintained pO2 levels in remnant kidneys (Priyadarshi et al., 2002). In humans, this hypothesis has not been confirmed so far; one study reported no correlation between R2* values and the degree of fibrosis (Ries et al., 2003), another found higher R2* values at increasing degrees of biopsy-proven fibrosis (Inoue et al., 2011). Finally, it is actually unknown whether CKD patients with high $\mathrm{R} 2 *$ values have more progressive disease than those with lower R2* values, and longitudinal follow-up studies are therefore eagerly awaited.

\section{BOLD-MRI AND DRUGS}

As stated in the introduction, BOLD-MRI can be repeated many times without side effects, making it an interesting tool to study the effect of drugs. On this behalf, the loop diuretic furosemide has been the most investigated. Blocking the $\mathrm{Na}^{+}-\mathrm{K}^{+}-2 \mathrm{Cl}^{-}$ transporter in the thick ascending loop of Henle with an IV bolus of furosemide leads to an acute decrease in active oxygenconsuming sodium transport, and increases local pO2 (Liss et al., 1999). As a proof of concept, acute furosemide-induced decreases in medullary R2* have been reported in both animals and humans (Brezis et al., 1994; Epstein and Prasad, 2000). Of interest, the medulla of CKD patients shows smaller decreases in $\mathrm{R} 2 *$ in response to furosemide than controls, and the furosemideinduced R2* change correlates well with the eGFR (Pruijm et al., 2014). This is explained by some as purely a dose effect due to the reduced renal function. Indeed, in order to exert its natriuretic action, furosemide has to be secreted by the proximal tubular cells, which is less the case at lower eGFR. Others see the $\mathrm{R} 2 *$ response as an indicator of tubular function and transport. For example, hypertensive individuals show, as CKD patients, a blunted R2* response to furosemide, although their eGFR is preserved, an observation that possibly refers to altered tubular sodium handling in hypertensives. Whether the $\mathrm{R} 2 *$ response to furosemide is indeed a marker of tubular function and mass, and has prognostic potential to predict adverse renal outcome, is currently unknown.

Few studies have tested other drugs with regard to their effect on R2*. One of the earliest studies reported acute increases in medullary and cortical $\mathrm{R} 2 *$ after iodinated contrast products, which is in line with their well-known vasoconstrictive and nephrotoxic potential (Hofmann et al., 2006). Inversely, acute decreases in R2* (within $1 \mathrm{~h}$ ) have been described after the administration of blockers of the renin-angiotensin system in small studies that included hypertensive or CKD patients (Djamali et al., 2007; Siddiqi et al., 2014). This is in line with the nephroprotective properties of these drugs, that lower intraglomerular pressure, tubular sodium transport, local inflammation, and oxidative stress (Tocci and Volpe, 2011). In contrast, chronic intake of renin-angiotensin system inhibitors did not alter renal R2* values (Pruijm et al., 2013), although a small decrease in cortical R2* values was found in hypertensive patients after 2 months of aliskiren (Vakilzadeh et al., 2015). These studies show that acute alterations in R2* occur, but they also illustrate once more that chronic renal tissue oxygenation is rather tightly controlled. The acute, drug induced- alterations in R2* offer insights in the mechanism of action and possibly nephroprotective potential of drugs in a preclinical setting. BOLD-MRI can be combined with other MRI methods such as arterial spin labeling, capable of measuring localrenal blood flow, or dynamic contrast enhanced-imaging that can directly measure glomerular filtration rate (Ebrahimi et al., 2014). Therefore, functional MRI can provide a wealth of information in the renal mechanisms of action of drugs, and possibly indicate renal side effects or benefits long before clinical outcome studies. Considering animal studies, functional MRI also has the potential to reduce animal usage. For these reasons, we expect functional MRI to play an increasingly important role in drug research.

\section{BOLD-MRI AND RENAL ARTERY STENOSIS}

As any organ, renal tissue oxygenation depends not only of local oxygen consumption (mainly active tubular sodium-dependent transport), but also of oxygen delivery (renal blood flow and hemoglobin level). Renal artery stenosis (RAS) is the classic example of ischemic nephropathy, and one would expect to find cortical and medullary hypoxia in this patient group. Animal 
studies have indeed reported acute increases in R2* after clipping of the renal artery (Juillard et al., 2004). However, no hypoxia could be detected with BOLD-MRI 4 weeks after clipping of the renal artery (Rognant et al., 2010). As in the remnant kidney model, the kidneys at the side of the clipped renal artery were atrophic and non-functional, and this study therefore does not necessarily reflect the situation as often encountered in the clinic when RAS kidneys are (slightly) reduced in size, but not atrophic.

In humans, BOLD-MRI has, to the best of our knowledge, not been used in situations of acute renal artery occlusion. In chronic RAS, increases in R2* have been reported in patients with severe RAS (>90\%), but not in those with less severe RAS (Gloviczki et al., 2011a). Hence, renal tissue oxygenation seems to be relatively independent from global renal blood flow. Of note, kidneys have the particularity that increases in RBF do not necessarily increase local pO2. Indeed, an increase in RBF leads, at constant filtration fraction, also to a higher GFR which on its term will increase the amount of filtered sodium, and thus the tubular sodium load and oxygen consumption (Hansell et al., 2013).

Some investigators have used the $\mathrm{R} 2^{*}$ response to furosemide as a marker of viability of renal tissue (Gloviczki et al., 2011b). In unilateral RAS, the eGFR does not provide information on the relative function of each kidney, whereas BOLD-MRI allows per-kidney analysis. The research group from the Mayo Clinic has demonstrated that the change in R2* after IV furosemide is smaller in those with severe RAS and reduced renal volume than in those with moderate RAS, and that non-viable renal tissue is therefore possibly defined as tissue that does not exhibit any change in R2* after furosemide (Gloviczki et al., 2011a).

\section{CLINICAL APPLICATIONS AND PERSPECTIVES}

\section{Renal Artery Stenosis}

Large randomized trials such as the Angioplasty and Stenting for Renal Artery Lesions trial (ASTRAL) have tempered the enthusiasm of clinicians to perform renal artery angioplasty \pm stenting in RAS patients (Investigators et al., 2009). Nevertheless, it is well known that some patients definitely benefit from angioplasty, and the main question remains how to predict the outcome of this procedure. The studies outlined above show that renal oxygenation can be maintained over a wide range of stenosis, but that above a certain individual threshold, chronic ischemia occurs, leading to inflammation, and decline of renal function, and diminished response to furosemide (Gloviczki et al., 2011a,b). This suggests that patients who will most likely benefit from angioplasty present chronic ischemia and a maintained response to furosemide, but this has not been tested in clinical practice. Hermann et al. recently reported that fractional hypoxia was higher in the stenotic kidneys of patients with high-grade RAS than in patients with essential hypertension (22.1 \pm 20 vs. $9.6 \pm 7 \%$ ). Fractional hypoxia diminished (from $22.1 \pm 20$ to $14.9 \pm 18.3 \%$ ) after renal stenting (Herrmann et al., 2016). In a similar way, an English research group showed that those with higher overall R2* levels and preserved renal volume and function had favorable outcomes after revascularization (Chrysochou et al., 2012). The integration of functional BOLDMRI in the workup and decision process of RAS opens new perspectives, but this needs further validation in clinical studies.

\section{Chronic Kidney Disease}

Ideally, BOLD-MRI should identify patients at increased risk of CKD progression, expecting that those with the lowest renal oxygenation, or the lowest R2* change after furosemide, have the highest risk of progression. So far, these data are lacking, hampering the introduction of BOLD-MRI in clinical practice.

Another issue is the fact that BOLD-MRI alone cannot establish whether a high $\mathrm{R}^{*}$ value is the result of low oxygen delivery (low RBF, extended renal fibrosis), high oxygen consumption (glomerular hyperfiltration, enhanced tubular absorption), or both. CKD is characterized by increased fibrosis, which on the one hand limits oxygen diffusion out of capillaries into renal cells, yet on the other hand also limits oxygen consumption. Quantifying fibrosis and regional blood flow, in combination with BOLD-MRI, could therefore provide useful information. Apart from $\mathrm{T}_{1}$ mapping, so-called diffusionweighted MRI (DW-MRI) offers a non-invasive way to quantify renal fibrosis and microcirculation. DW-MRI collects images with and without diffusion weighted gradients, and expresses molecular diffusion as the apparent diffusion coefficient (ADC) Liss et al., 2013. The total ADC can be separated in a perfusion fraction $\left(\mathrm{F}_{\mathrm{p}}\right)$ and perfusion-free diffusion $\left(A D C_{D}\right)$, as measures of local microcirculation and fibrosis. A reduction in ADC has been shown to correlate with CKD staging and the degree of fibrosis (Xu et al., 2010; Zhao et al., 2014), and a reduction in $\mathrm{F}_{\mathrm{p}}$ has been demonstrated in renal allografts suffering from acute rejection (Eisenberger et al., 2010). Whether a combination of BOLD-MRI and diffusion MRI is capable of predicting renal function decline is not yet known, and subject of active research.

\section{CONCLUSION}

BOLD-MRI is an exciting technique to assess renal oxygenation non-invasively in humans. Increased knowledge of factors that influence the BOLD-signal has lead to better standardization, and refinements in the analysis technique to highly reproducible results. BOLD-MRI is a powerful tool to detect the influence of altered hemodynamics, drugs, or dietary factors on renal oxygenation. The near future will tell if BOLD-MRI (alone or in combination with other MRI modalities) allows the selection of RAS patients who will benefit from revascularization, and/or early identification of CKD patients at high risk for renal function decline.

\section{AUTHOR CONTRIBUTIONS}

MP: Drafting the article, final approval of the version to be published, agrees to be accountable for all aspects of the work in ensuring that questions related to the accuracy or integrity of any part of the work are appropriately investigated and resolved. BM and MB: Revising the article critically for important intellectual 
content, final approval of the submitted version, both agree to be accountable for all aspects of the work in ensuring that questions related to the accuracy or integrity of any part of the work are appropriately investigated and resolved.

\section{REFERENCES}

Alberti, K. G., and Zimmet, P. Z. (1998). Definition, diagnosis and classification of diabetes mellitus and its complications. Part 1: diagnosis and classification of diabetes mellitus provisional report of a WHO consultation. Diabetic medicine: a journal of the British Diabetic Association. 15, 539-553. doi: 10.1002/(SICI)1096-9136(199807)15:7<539::AID-DIA668>3.0.CO;2-S

Astor, B. C., Matsushita, K., Gansevoort, R. T., van der Velde, M., Woodward, M., Levey, A. S., et al. (2011). Lower estimated glomerular filtration rate and higher albuminuria are associated with mortality and end-stage renal disease. A collaborative meta-analysis of kidney disease population cohorts. Kidney Int. 79, 1331-1340. doi: 10.1038/ki.2010.550

Aukland, K., and Krog, J. (1960). Renal oxygen tension. Nature 188:671. doi: $10.1038 / 188671 \mathrm{a} 0$

Brezis, M., Agmon, Y., and Epstein, F. H. (1994). Determinants of intrarenal oxygenation. I. Effects of diuretics. Am. J. Physiol. 267, F1059-F1062.

Chrysochou, C., Mendichovszky, I. A., Buckley, D. L., Cheung, C. M., Jackson, A., and Kalra, P. A. (2012). BOLD imaging: a potential predictive biomarker of renal functional outcome following revascularization in atheromatous renovascular disease. Nephrol. Dial Transplant. 27, 1013-1019. doi: $10.1093 / \mathrm{ndt} / \mathrm{gfr} 392$

Djamali, A., Sadowski, E. A., Muehrer, R. J., Reese, S., Smavatkul, C., Vidyasagar, A., et al. (2007). BOLD-MRI assessment of intrarenal oxygenation and oxidative stress in patients with chronic kidney allograft dysfunction. Am. J. Physiol. Renal Physiol. 292, F513-F522. doi: 10.1152/ajprenal.00222.2006

Ebrahimi, B., Textor, S. C., and Lerman, L. O. (2014). Renal relevant radiology: renal functional magnetic resonance imaging. Clin. J. Am. Soc. Nephrol. 9, 395-405. doi: 10.2215/CJN.02900313

Eisenberger, U., Thoeny, H. C., Binser, T., Gugger, M., Frey, F. J., Boesch, C., et al. (2010). Evaluation of renal allograft function early after transplantation with diffusion-weighted MR imaging. Eur. Radiol. 20, 1374-1383. doi: 10.1007/s00330-009-1679-9

Epstein, F. H., and Prasad, P. (2000). Effects of furosemide on medullary oxygenation in younger and older subjects. Kidney Int. 57, 2080-2083. doi: 10.1046/j.1523-1755.2000.00057.x

Fine, L. G., and Norman, J. T. (2008). Chronic hypoxia as a mechanism of progression of chronic kidney diseases: from hypothesis to novel therapeutics. Kidney Int. 74, 867-872. doi: 10.1038/ki.2008.350

Gloviczki, M. L., Glockner, J. F., Crane, J. A., McKusick, M. A., Misra, S., Grande, J. P., et al. (2011a). Blood oxygen level-dependent magnetic resonance imaging identifies cortical hypoxia in severe renovascular disease. Hypertension 58, 1066-1072. doi: 10.1161/HYPERTENSIONAHA.111.171405

Gloviczki, M. L., Lerman, L. O., and Textor, S. C. (2011b). Blood oxygen leveldependent (BOLD) MRI in renovascular hypertension. Curr. Hypertens. Rep. 13, 370-377. doi: 10.1007/s11906-011-0218-7

Hansell, P., Welch, W. J., Blantz, R. C., and Palm, F. (2013). Determinants of kidney oxygen consumption and their relationship to tissue oxygen tension in diabetes and hypertension. Clin. Exp. Pharmacol. Physiol. 40, 123-137. doi: 10.1111/1440-1681.12034

Herrmann, S. M., Saad, A., Eirin, A., Woollard, J., Tang, H., McKusick, M. A., et al. (2016). Differences in GFR and tissue oxygenation, and interactions between stenotic and contralateral kidneys in unilateral atherosclerotic renovascular disease. Clin. J. Am. Soc. Nephrol. 11, 458-469. doi: 10.2215/CJN.036 20415

Heyman, S. N., Khamaisi, M., Rosen, S., and Rosenberger, C. (2008). Renal parenchymal hypoxia, hypoxia response and the progression of chronic kidney disease. Am. J. Nephrol. 28, 998-1006. doi: 10.1159/000146075

Hofmann, L., Simon-Zoula, S., Nowak, A., Giger, A., Vock, P., Boesch, C., et al. (2006). BOLD-MRI for the assessment of renal oxygenation in humans: acute effect of nephrotoxic xenobiotics. Kidney Int. 70, 144-150. doi: $10.1038 /$ sj.ki. 5000418

\section{FUNDING}

This work was supported by grants from the Swiss National Science Fondation (FN 32003B-149309 and 320030-169191).

Inoue, T., Kozawa, E., Okada, H., Inukai, K., Watanabe, S., Kikuta, T., et al. (2011). Noninvasive evaluation of kidney hypoxia and fibrosis using magnetic resonance imaging. J. Am. Soc. Nephrol. 22, 1429-1434. doi: 10.1681/ASN.2010111143

Investigators, A., Wheatley, K., Ives, N., Gray, R., Kalra, P. A., Moss, J. G., et al. (2009). Revascularization versus medical therapy for renal-artery stenosis. $N$. Engl. J. Med. 361, 1953-1962. doi: 10.1056/NEJMoa0905368

Juillard, L., Lerman, L. O., Kruger, D. G., Haas, J. A., Rucker, B. C., Polzin, J. A., et al. (2004). Blood oxygen level-dependent measurement of acute intra-renal ischemia. Kidney Int. 65, 944-950. doi: 10.1111/j.1523-1755.2004.00469.x

Khatir, D. S., Pedersen, M., Jespersen, B., and Buus, N. H. (2015). Evaluation of renal blood flow and oxygenation in CKD using magnetic resonance imaging. Am. J. Kidney Dis. 66, 402-411. doi: 10.1053/j.ajkd.2014.11.022

Liss, P., Cox, E. F., Eckerbom, P., and Francis, S. T. (2013). Imaging of intrarenal haemodynamics and oxygen metabolism. Clin. Exp. Pharmacol. Physiol. 40, 158-167. doi: 10.1111/1440-1681.12042

Liss, P., Nygren, A., Ulfendahl, H. R., and Erikson, U. (1999). Effect of furosemide or mannitol before injection of a non-ionic contrast medium on intrarenal oxygen tension. Adv. Exp. Med. Biol. 471, 353-359. doi: 10.1007/978-1-4615-4717-4_42

Milani, B., Ansaloni, A., Sousa-Guimaraes, S., Piskunowicz, M., Vogt, B., Stuber, M., et al. (2016). [Op.4d.06] reduction of cortical oxygenation in chronic kidney disease: evidence obtained with bold-mri and a new analytic technique. $J$. Hypertens. 34(Suppl. 2):e52. doi: 10.1097/01.hjh.0000491472.37273.d4

Neugarten, J. (2012). Renal BOLD-MRI and assessment for renal hypoxia. Kidney Int. 81, 613-614. doi: 10.1038/ki.2011.462

Pedersen, M., Dissing, T. H., Mørkenborg, J., Stødkilde-Jorgensen, H., Hansen, L. H., Pedersen, L. B., et al. (2005). Validation of quantitative BOLD MRI measurements in kidney: application to unilateral ureteral obstruction. Kidney Int. 67, 2305-2312. doi: 10.1111/j.1523-1755.2005.00334.x

Piskunowicz, M., Hofmann, L., Zuercher, E., Bassi, I., Milani, B., Stuber, M., et al. (2015). A new technique with high reproducibility to estimate renal oxygenation using BOLD-MRI in chronic kidney disease. Magn. Reson. Imaging 33, 253-261. doi: 10.1016/j.mri.2014.12.002

Ponte, B., Pruijm, M., Marques-Vidal, P., Martin, P. Y., Burnier, M., Paccaud, F., et al. (2013). Determinants and burden of chronic kidney disease in the population-based CoLaus study: a cross-sectional analysis. Nephrol. Dial. Transplant. 28, 2329-2339. doi: 10.1093/ndt/gft206

Prasad, P. V. (2006). Evaluation of intra-renal oxygenation by BOLD MRI. Nephron Clin. Pract. 103, c58-c65. doi: 10.1159/000090610

Prasad, P. V., Edelman, R. R., and Epstein, F. H. (1996). Noninvasive evaluation of intrarenal oxygenation with BOLD MRI. Circulation 94, 3271-3275. doi: 10.1161/01.CIR.94.12.3271

Prasad, P. V., and Epstein, F. H. (1999). Changes in renal medullary $\mathrm{pO}_{2}$ during water diuresis as evaluated by blood oxygenation level-dependent magnetic resonance imaging: effects of aging and cyclooxygenase inhibition. Kidney Int. 55, 294-298. doi: 10.1046/j.1523-1755.1999.00237.x

Prasad, P. V., Thacker, J., Li, L. P., Haque, M., Li, W., Koenigs, H., et al. (2015). Multi-parametric evaluation of chronic kidney disease by mri: a preliminary cross-sectional study. PLOS ONE 10:e0139661. doi: 10.1371/journal.pone.0139661

Priyadarshi, A., Periyasamy, S., Burke, T. J., Britton, S. L., Malhotra, D., and Shapiro, J. I. (2002). Effects of reduction of renal mass on renal oxygen tension and erythropoietin production in the rat. Kidney Int. 61, 542-546. doi: 10.1046/j.1523-1755.2002.00140.x

Pruijm, M., Hofmann, L., Maillard, M., Tremblay, S., Glatz, N., Wuerzner, G., et al. (2010). Effect of sodium loading/depletion on renal oxygenation in young normotensive and hypertensive men. Hypertension 55, 1116-1122. doi: 10.1161/HYPERTENSIONAHA.109.149682

Pruijm, M., Hofmann, L., Piskunowicz, M., Muller, M. E., Zweiacker, C., Bassi, I., et al. (2014). Determinants of renal tissue oxygenation as measured with 
BOLD-MRI in chronic kidney disease and hypertension in humans. PLoS ONE 9:e95895. doi: 10.1371/journal.pone.0095895

Pruijm, M., Hofmann, L., Zanchi, A., Maillard, M., Forni, V., Muller, M. E., et al. (2013). Blockade of the renin-angiotensin system and renal tissue oxygenation as measured with BOLD-MRI in patients with type 2 diabetes. Diabetes Res. Clin. Pract. 99, 136-144. doi: 10.1016/j.diabres.2012.11.004

Ries, M., Basseau, F., Tyndal, B., Jones, R., Deminiere, C., Catargi, B., et al. (2003). Renal diffusion and BOLD MRI in experimental diabetic nephropathy. Blood oxygen level-dependent. J. Magn. Reson. Imaging 17, 104-113. doi: 10.1002/jmri.10224

Rognant, N., Rouviere, O., Janier, M., Le, Q. H., Barthez, P., Laville, M., et al. (2010). Hemodynamic responses to acute and gradual renal artery stenosis in pigs. Am. J. Hypertens. 23, 1216-1219. doi: 10.1038/ajh.2010.147

Saad, A., Crane, J., Glockner, J. F., Herrmann, S. M., Friedman, H., Ebrahimi, B., et al. (2013). Human renovascular disease: estimating fractional tissue hypoxia to analyze blood oxygen level-dependent MR. Radiology 268, 770-778. doi: 10.1148/radiol.13122234

Siddiqi, L., Hoogduin, H., Visser, F., Leiner, T., Mali, W. P., and Blankestijn, P. J. (2014). Inhibition of the renin-angiotensin system affects kidney tissue oxygenation evaluated by magnetic resonance imaging in patients with chronic kidney disease. J. Clin. Hypertens. 16, 214-218. doi: 10.1111/jch.12263

Simon-Zoula, S. C., Hofmann, L., Giger, A., Vogt, B., Vock, P., Frey, F. J., et al. (2006). Non-invasive monitoring of renal oxygenation using BOLD-MRI: a reproducibility study. NMR Biomed. 19, 84-89. doi: 10.1002/nbm.1004
Tocci, G., and Volpe, M. (2011). End-organ protection in patients with hypertension: focus on the role of angiotensin receptor blockers on renal function. Drugs 71, 1003-1017. doi: 10.2165/11591350-000000000-00000

Vakilzadeh, N., Muller, M. E., Forni, V., Milani, B., Hoffman, L., Piskunowicz, M., et al. (2015). Comparative effect of a renin inhibitor and a thiazide diuretic on renal tissue oxygenation in hypertensive patients. Kidney Blood Press. Res. 40, 542-554. doi: 10.1159/000368530

Xu, X., Fang, W., Ling, H., Chai, W., and Chen, K. (2010). Diffusion-weighted MR imaging of kidneys in patients with chronic kidney disease: initial study. Eur. Radiol. 20, 978-983. doi: 10.1007/s00330-009-1619-8

Zhao, J., Wang, Z. J., Liu, M., Zhu, J., Zhang, X., Zhang, T., et al. (2014). Assessment of renal fibrosis in chronic kidney disease using diffusion-weighted MRI. Clin. Radiol. 69, 1117-1122. doi: 10.1016/j.crad.2014.06.011

Conflict of Interest Statement: The authors declare that the research was conducted in the absence of any commercial or financial relationships that could be construed as a potential conflict of interest.

Copyright (c) 2017 Pruijm, Milani and Burnier. This is an open-access article distributed under the terms of the Creative Commons Attribution License (CC BY). The use, distribution or reproduction in other forums is permitted, provided the original author(s) or licensor are credited and that the original publication in this journal is cited, in accordance with accepted academic practice. No use, distribution or reproduction is permitted which does not comply with these terms. 